\title{
Aplicação do CRS-parcial em dados esparsos para a inversão NIP-tomográfica.
}

Felipe A. V. Pena, Lourenildo W. B. Leite e Wildney W. S. Vieira, UFPA, Brasil.

Copyright 2014, SBGf - Sociedade Brasileira de Geofísica.

Este texto foi preparado para a apresentação no VI Simpósio Brasileiro de Geofísica Porto Alegre, 14 a 16 de outubro de 2014. Seu conteúdo foi revisado pelo Comitê Técnico do VI SimBGf, mas não necessariamente representa a opinião da SBGf ou de seus associados. É proibida a reprodução total ou parcial deste material para propósitos comerciais sem prévia autorização da SBGf.

\section{Resumo}

Este trabalho teve como objetivo a aplicação de métodos do empilhamento superfície-de-reflexãocomum (CRS) convencional, do CRS-parcial e da inversão NIP-tomográfica para gerar imagens sísmicas em dados sísmicos esparsos. Para analisar o resultado do método aplicado, foram realizados testes com dados sintéticos esparsos pelo silenciamento aleatório de traços nas famílias CMP. Em continuação, foi analisado o efeito do empilhamento na obtenção do modelo de velocidade em profundidade pela inversão (não-linear, multiparamétrica) niptomográfica que utiliza para vínculo os atributos cinemáticos do campo de onda para estimar um modelo coerente com o dado.

\section{Introdução}

A qualidade do dado de reflexão sísmica, medida pela alta relação $S / R$, é uma característica importante para o processamento sísmico. Pena (2014) analisou o efeito do espaçamento não uniforme e esparso que pode resultar num prejuízo para a análise de velocidade aplicada, e sua aplicação no empilhamento e no imageamento da seção. A interpolação de dados nas seções pelo preenchimento de lacunas pode ser realizado normalmente por diferentes técnicas.

Müller (1999) descreveu o método CRS, onde a superfície de empilhamento aproxima o tempo de trânsito primário para o evento sísmico de forma mais precisa e consistente do que o operador NMO/DMO.

Baykulov and Gajewski (2007) apresentou uma consequência do operador CRS na interpolação, chamada de CRS-parcial, gerando superfamílias, onde lacunas são preenchidas, e melhorada a relação $S / R$ das seções pré-empilhadas, empilhadas e migradas. Com isto, resulta em seções onde a frequência espacial de Nyquist, $f_{N}=\frac{1}{2 \Lambda x}$, é estendida, e a contaminação por amostragem muito diminuída.

Duveneck (2004) apresentou a inversão NIP-tomográfica para se obter uma distribuição de velocidades para o imageamento em profundidade, onde vínculos são fortes e estabelecidos pelos atributos CRS obtidos no processo de empilhamento.

\section{Empilhamento CRS}

O operador CRS dado pela equação (1) depende das coordenadas espaciais deslocamento do ponto-médio $\left(x_{m}-x_{0}\right)$ e meio-afastamento $(h)$, e o processo produz uma seção simulada ao afastamento-nulo. No processo são estimados os atributos de frente de onda: ângulo de emergência $\left(\alpha_{0}\right)$, o raio de curvatura da onda NIP $\left(R_{\mathrm{NIP}}\right)$, e o raio de curvatura da onda $\mathrm{N}\left(R_{\mathrm{N}}\right)$ relativos ao ponto de referência $P_{0}\left(x_{0}, t_{0}\right)$.

$$
t^{2}\left(x_{m}, h\right)=\left(t_{0}+2 \frac{\sin \alpha_{0}}{v_{0}}\left(x_{m}-x_{0}\right)\right)^{2}+2 t_{0} \frac{\cos ^{2} \alpha_{0}}{v_{0}}\left(\frac{\left(x_{m}-x_{0}\right)^{2}}{R_{\mathrm{N}}}+\frac{h^{2}}{R_{\mathrm{NIP}}}\right) .
$$

A Figura 1 exemplifica o CRS-parcial, que é baseado numa superfície limitada para o empilhamento ao redor de um ponto-médio, e tempo de trânsito escolhido, $P_{0}\left(x_{0}, t_{0}\right)$, e o resultado da soma gera a amplitude de uma nova amostra. Repetindo este processo para todos os pontos temporais ao longo do traço, e para todos os CMP estabelecidos para a interpolação, produz a nova família CRS-superfamília.

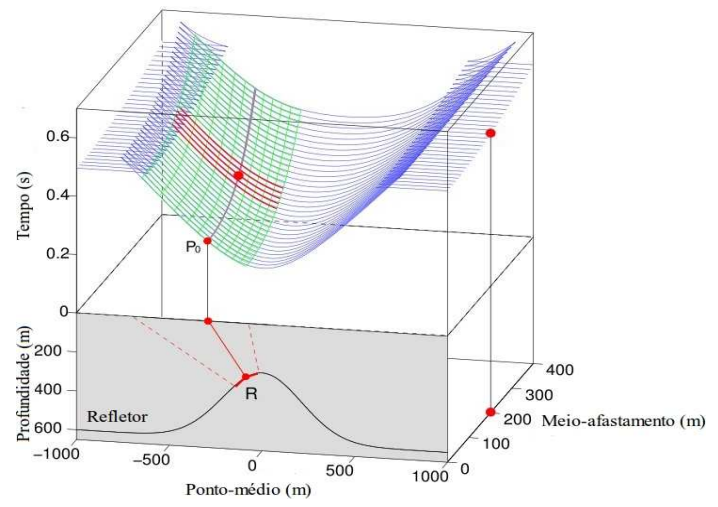

Figura 1: A superfície verde é do CRS convencional, que atribui o resultado ao ponto $P_{0}$. A superfície vermelha é do CRS-parcial, e que coincide localmente com a superfície do CRS convencional. O CRS-parcial preenche a lacuna no ponto central da superfície vermelha.

A estimativa dos parâmetros do operador CRS (1) é realizada através de estratégias que utilizam casos particulares desta equação do tempo de trânsito. A busca tri-paramétrica é realizada no domínio do semblance que é desdobrada em problemas mais simples que envolvem apenas um parâmetro desconhecido como no caso NMO.

A Figura 2 ilustra o cálculo do tempo $t_{0}^{\prime}$ do evento $A$ no CRS-parcial, onde se usa a média dos atributos do CRS convencional. A amplitude é estimada como a média ao longo da superfície estabelecida.

O tempo $t_{0}$ exato que se ajusta perfeitamente na hipérbole do evento em questão é calculado pela equação (2) usando os parâmetros CRS utilizados na aproximação do tempo de trânsito $t_{0}^{\prime}$, esta equação é derivada de (1) após resolver a equação quadrática para $x_{m}=0$, considerando 
apenas valores positivos,

$$
t_{0}=-\frac{h_{A}^{2} \cos ^{2} \alpha}{V_{0} R_{\mathrm{NIP}}}+\sqrt{\left(\frac{h_{A}^{2} \cos ^{2} \alpha}{v_{0} R_{N I P}}\right)^{2}+t_{A}^{2}} .
$$

A equação (3) calcula o tempo de trânsito da superfície do CRS-parcial, que utiliza os parâmetros correspondentes a $t_{0}^{\prime}$, e que é obtida substituindo $t_{0}$ da equação (2) na equação (1):

$$
\begin{aligned}
t^{2}\left(x_{m}, h\right)= & \left(-\frac{h_{A}^{2} \cos ^{2} \alpha}{v_{0} R_{N I P}}+\sqrt{\left(\frac{h_{A}^{2} \cos ^{2} \alpha}{v_{0} R_{\mathrm{NIP}}}\right)^{2}+t_{A}^{2}}+\frac{2 \sin \alpha}{v_{0}}\left(x_{m}-x_{0}\right)\right)^{2} \\
+ & \frac{2 \cos ^{2} \alpha}{v_{0}}\left(-\frac{h_{A}^{2} \cos ^{2} \alpha}{v_{0} R_{\mathrm{NIP}}}+\sqrt{\left(\frac{h_{A}^{2} \cos ^{2} \alpha}{v_{0} R_{\mathrm{NIP}}}\right)^{2}+t_{A}^{2}}\right) \\
& \left(\frac{\left(x_{m}-x_{0}\right)^{2}}{R_{\mathrm{N}}}+\frac{h_{A}^{2}}{R_{\mathrm{NIP}}}\right) .
\end{aligned}
$$

Ao longo desta superfície as amplitudes são somadas, e o resultado divido pelo numero de traços envolvidos na somatória 2D, gerando assim um traço para compor uma superfamília CRS.

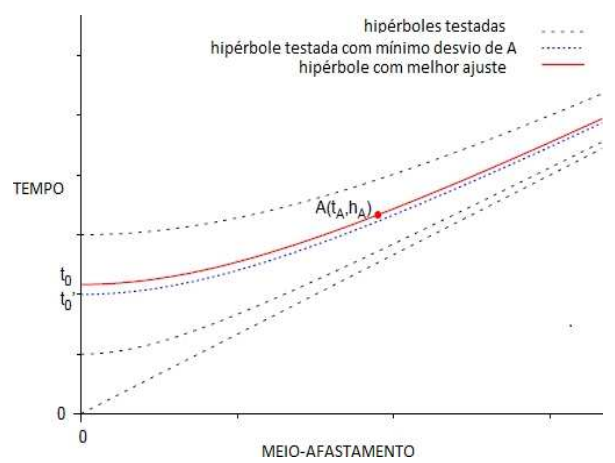

Figura 2: Curvas de tempos de trânsitos testados para o melhor ajuste da hipérbole $A\left(t_{A}, h_{A}\right)$.

\section{Inversão}

A inversão tomográfica da onda NIP associada a cada ponto $P_{0}\left(t_{0}, \xi_{0}\right)$ é organizada com o tempo de trânsito do raio normal, $\tau_{0}=\frac{t_{0}}{2}$, pela posição de emergência, $\xi_{0}$, do raio normal, pelo parâmetro horizontal do raio, $p^{(\xi)}$, e pela derivada espacial de segunda ordem do tempo de trânsito, $M_{\mathrm{NIP}}^{(\xi)}$. Estes dados de entrada são estimados a partir dos atributos CRS, e são organizado no vetor de entrada dado por:

$$
\mathbf{d}_{\mathrm{obs}}=\left(\tau_{0}, M_{\mathrm{NIP}}^{(\xi)}, p^{(\xi)}, \xi_{0}\right)_{i=1}^{n},
$$

onde $n$ é o número de pontos marcados na seção CRS empilhada.

O modelo de velocidade é definido em uma malha com $n_{x}$ e $n_{z}$ nós na horizontal e vertical, respectivamente, e dado por:

$$
v(x, z)=\sum_{j=1}^{n_{x}} \sum_{k=1}^{n_{z}} v_{i j} \beta_{j}(x) \beta_{k}(-z),
$$

onde $\beta_{j}$ e $\beta_{k}$ são as funções de bases B-splines, e $v_{i j}$ são os coeficientes a serem determinados no processo de inversão.
A Figura 3 exemplifica a tomografia da onda NIP 2D em subsuperfície, ponto $(x, z)$, e pela direção do raio normal dado ângulo de inclinação local $\theta$. Sendo assim, o problema para estimar o modelo de velocidade consiste em achar as componentes do vetor $\mathbf{m}$ definido por:

$$
\begin{aligned}
(x, z, \theta)_{i}^{(\mathrm{NIP})}, & \left(i=1, \ldots, n_{\text {dados }}\right), \\
v_{j k}, & \left(j=1, \ldots, n_{x}, k=1, \ldots, n_{z}\right) .
\end{aligned}
$$

Sendo assim, existem $\mathrm{N}_{\text {dados }}=4 n_{\text {dados }}$ dados de entrada, e $\mathrm{M}_{\text {par }}=3 n_{\text {dados }}+n_{x} n_{z}$ parâmetros a serem determinados.

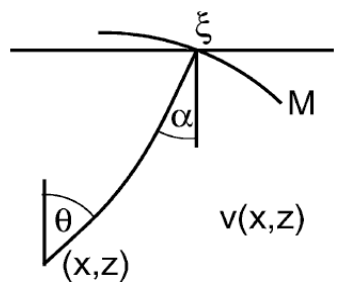

Figura 3: Componentes dos dados de entrada para a inversão contidos no vetor (4), e no modelo (6) para a inversão tomográfica da onda NIP.

O problema de inversão é ajuste de curvas, e consiste na busca de um modelo ótimo $\mathbf{m}$ tal que o desvio entre o dado observado, $\mathbf{d}_{\mathrm{obs}}$, e o correspondente teórico, $\mathbf{d}_{\bmod }=$ $f(m)$, seja minimizado no sentido dos quadrados-mínimos quadrados dos desvios $\Delta \mathbf{d}(\mathbf{m})=\mathbf{d}-\mathbf{f}(\mathbf{m})$, considerando uma $\mathbf{C}_{D}$ matriz simétrica para a covariância dos dados, o que implica em erros não-correlacionáveis.

A série de Taylor é a técnica usada para linearizar a função desajuste na vizinhança de, $\mathbf{m}_{n}$, e dada por:

$$
f\left(\mathbf{m}_{n}+\Delta \mathbf{m}\right) \approx f\left(\mathbf{m}_{n}\right)+\mathbf{F} \Delta \mathbf{m},
$$

onde $\mathbf{F}$ é a matriz das derivadas $F_{i j}=\frac{\partial f_{i}}{\partial m_{j}}$, de $\mathbf{f}$ no ponto $\mathbf{m}_{n}$.

Regularização é um conceito central em inversão, e as diferentes formas de regularização introduzidas estabelecem uma nova solução ao problema. A condição de suavidade é também necessária para assegurar a validação da teoria paraxial do raio ao redor de cada raio central, permitindo relaciona-la ao cálculo dos atributos CRS. Sendo assim, a função objeto de minimização é reescrita na forma:

$$
\Phi(\mathbf{m})=\frac{1}{2} \Delta \mathbf{d}^{T}(\mathbf{m}) \mathbf{C}_{D}^{-1} \Delta \mathbf{d}(\mathbf{m})+\frac{1}{2} \varepsilon^{\prime \prime} \mathbf{m}^{(v) T} \mathbf{D}^{\prime \prime} \mathbf{m}^{(v)},
$$

com o vetor dos parâmetros alterado para,

$$
\mathbf{m}=\left(\begin{array}{c}
\mathbf{m}^{(\mathrm{NIP})} \\
\mathbf{m}^{(v)}
\end{array}\right)
$$

A matriz $\mathbf{D}^{\prime \prime}$ é positiva definida, e representa a parte da regularização com a segunda derivada espacial para os parâmetros, o que penaliza a rugosidade do modelo. A quantidade $\varepsilon^{\prime \prime}$ pondera a participação entre os termos desajuste e regularização.

A aplicação do gradiente $\nabla_{\mathbf{m}} \Phi(\mathbf{m})=\mathbf{0}$ na equação (8), é a condição para a minimização, e resulta nas equações 
normais, de onde se constrói o sistema matricial para $\hat{\mathbf{F}}$ e para o vetor $\Delta \hat{\mathbf{d}}$ nas formas:

$$
\hat{\mathbf{F}}=\left(\begin{array}{c}
\mathbf{C}_{D}^{-1 / 2} \mathbf{F} \\
\tilde{\mathbf{B}}
\end{array}\right) \text { e } \Delta \hat{\mathbf{d}}=\left(\begin{array}{c}
\mathbf{C}_{D}^{-1 / 2} \Delta \mathbf{d}\left(\mathbf{m}_{n}\right) \\
\tilde{\mathbf{B}} \mathbf{m}_{n}
\end{array}\right) ;
$$

Com isto, o sistema a ser resolvido é reescrito na forma compacta dada por:

$$
\hat{\mathbf{F}}^{T} \hat{\mathbf{F}} \Delta \mathbf{m}=\hat{\mathbf{F}}^{T} \Delta \hat{\mathbf{d}} .
$$

A matriz $\tilde{\mathbf{B}}$ tem por definição que $\tilde{\mathbf{B}}^{T} \tilde{\mathbf{B}}=\varepsilon^{\prime \prime} \tilde{\mathbf{D}}^{\prime \prime}$. As linhas da matriz $\tilde{\mathbf{B}}$ em $\mathbf{F}$ e $\Delta \hat{\mathbf{d}}$, que correspondem aos parâmetros NIP do modelo, são nulas.

Paige and Saunders (1982) apresentaram o algorítmo LSQR para a solução da equação (11), com um método eficiente para resolver sistemas lineares. O vetor modelo para a a iteração sucessiva é atualizado na forma:

$$
\mathbf{m}_{n+1}=\mathbf{m}_{n}+\lambda \Delta \mathbf{m}_{\mathrm{n}} ;
$$

onde $\lambda \leq 1$ é o fator de amortização dos passos $\Delta \mathbf{m}$.

Durante as iterações os valores $\mathbf{m}_{n}$ são obtidos, e novos dados são calculados pelo traçamento dinâmico do raio. Se a função objeto de minimização em (8) aumenta, $\lambda$ é diminuído, então $\Phi(\mathbf{m})$ é recalculada. Do contrário, a próxima iteração é iniciada calculando as derivadas de Fréchet para o novo modelo.

\section{Resultados e Conclusões}

Os experimentos, buscando a otimização e a sistematização para o empilhamento, seguido da inversão NIP-tomográfica ao modelo em profundidade, para o caso de dados sísmicos esparsos, foram organizados em três partes: CMP; CRS-convencional; e CRS-superfamília.

A figura 4 exibe o modelo sintético construído para simular o ambiente geológico, que foi baseado na descrição de Duveneck (2004). O modelo consiste em camadas homogêneas e isotrópicas limitadas por interfaces curvas suaves, com velocidades que varia de $2000 \mathrm{~m} / \mathrm{s}$ no topo, a $5000 \mathrm{~m} / \mathrm{s}$ na parte mais inferior. O modelo geológico deve satisfazer as exigências do modelo teórico, que é a teoria paraxial do raio sísmico.

Os experimentos com dados esparsos foram realizados com a eliminação drástica e aleatória de $80 \%$ dos traços da seção utilizando o programa sukill. O dado esparsado foi utilizado como entrada para a busca automática dos atributos do campo de onda, com uma abertura para o CRS-parcial de $-1475 \mathrm{a}+1475$ na dimensão afastamento, e de $200 \mathrm{~m}$ no tempo de trânsito de $0.3 \mathrm{~s}$ à $300 \mathrm{~m}$ em $2.7 \mathrm{~s}$ na coordenada ponto-médio.

A malha construída $\left(n_{x} \times n_{z}\right)$ para os experimentos foi definida com $(23 \times 16)$ nós, e os fatores de ponderação com $\varepsilon_{\mathrm{xx}}=0.004$ e $\varepsilon_{\mathrm{zz}}=0.0002$.

Como um exemplo, a figura 5 mostra o resultado na família CMP 200 antes e depois o empilhamento CRS-parcial. Observa-se como a relação $S / R$ aumenta, e com isto a visualização dos eventos sísmicos é proeminente.

O empilhamento CRS-parcial tem dupla ação: gera uma nova família CRS que pode ser aumentada; interpola traços para preencher lacunas na família original. Com isto, permite estatisticamente melhora a relação $S / R$.

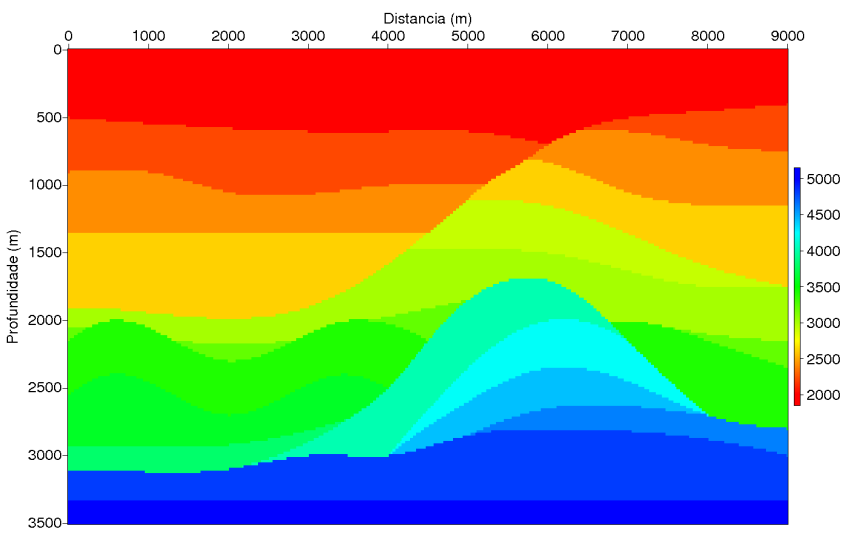

Figura 4: Modelo de velocidade em bloco.
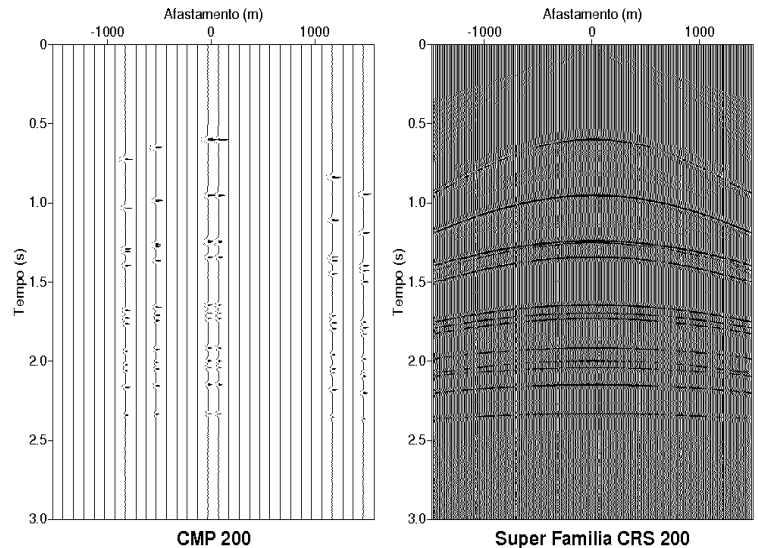

Super Familia CRS 200

Figura 5: Família CMP 200 antes e depois do empilhamento CRS-parcial.

\section{Inversão: CMP}

A primeira parte foi referente ao empilhamento CMP usado como entrada na inversão NIP-tomográfica para obter o modelo de velocidade em profundidade. Foi realizado uma marcação automática que gera os dados observados de entrada a partir das seguintes seções: a empilhada CMP; a coerência semblance CMP; a $R_{N I P}$; e a ângulo de emergência $\alpha$. A coerência mínima para selecionar uma marcação (parâmetro mincoher) foi de 0,19 . O total de marcações foi 399, e reduzida a 395 pontos após edição.

A figura 6 mostra 0 resultado da coerência no empilhamento CMP com valor máximo ao redor de 0,35 .

A figura 7 mostra o resultado do empilhamento CMP, onde a presença de muitas lacunas nos eventos da seção empilhada pode ser observado.

A figura 8 mostra o modelo de velocidade obtido após 30 iterações utilizando um modelo inicial com velocidade constante, $v_{0}=2000 \mathrm{~m} / \mathrm{s}$. A marcação automática está distribuída sobre o resultado. Apesar do modelo inicial ser muito diferente do original, a NIP-tomografia foi capaz de reproduzir o aspecto geral do modelo de velocidade 
original da figura 4.

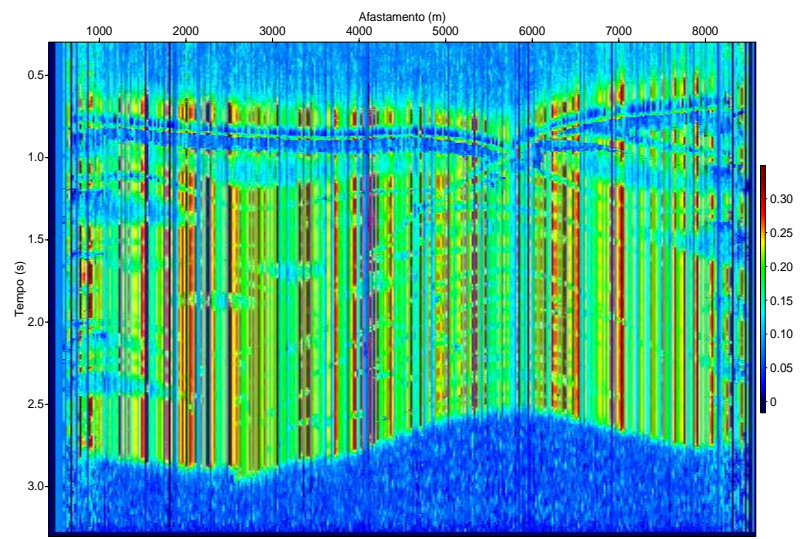

Figura 6: Seção coerência semblance resultante do empilhamento CMP-automático.

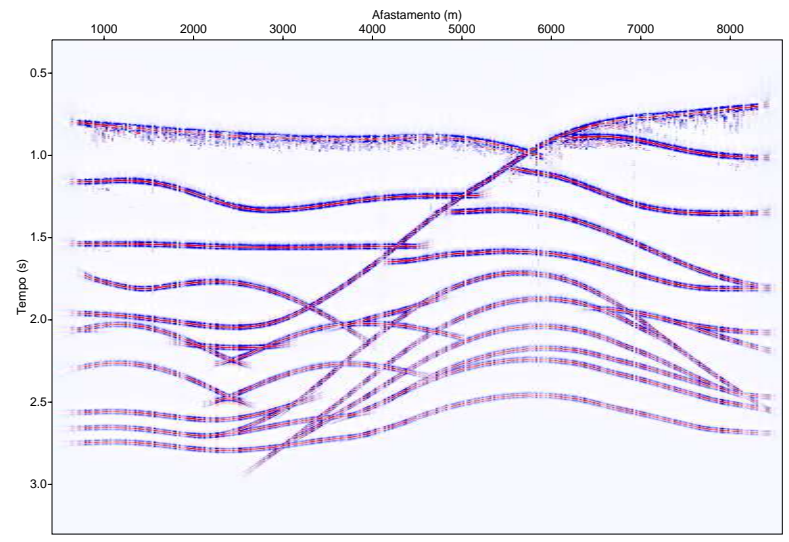

Figura 7: Seção empilhada CMP-automático

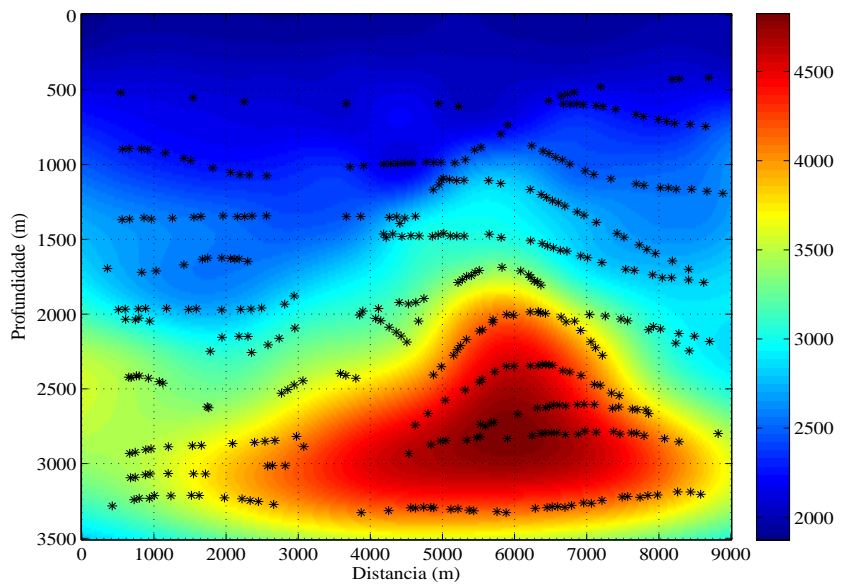

Figura 8: Modelo de velocidade resultante após 30 interações com as marcações da seção empilhada CMPautomática sobrepostas.

\section{Inversão: CRS-convencional}

A segunda parte foi referente ao empilhamento CRSconvencional usado como entrada na inversão NIPtomográfica para também se obter o modelo de velocidade em profundidade. As seções de dados de entrada para a marcação automática foram: a empilhada CRSoptimizada; a optimizada $R_{N I P}$; a ângulo de emergência $\alpha$; e a coerência semblance optimizada. Apesar de ter uma maior continuidade na coerência dos eventos, o valor de coerência máxima foi menor do que a resultante para o empilhamento CMP-automático. A coerência mínima válida para a marcação foi de 0,19 , e o total de marcações foi de 352 pontos.

A figura 9 mostra a seção coerência, onde o valor máximo está ao redor de 0,25 , e menor do que o caso CMP da primeira parte.

A figura 10 mostra o resultado do empilhamento CRSconvencional, o qual apresenta uma melhora considerável em relação à figura 7 , uma vez que quase todas as lacunas foram preenchidas no empilhamento.

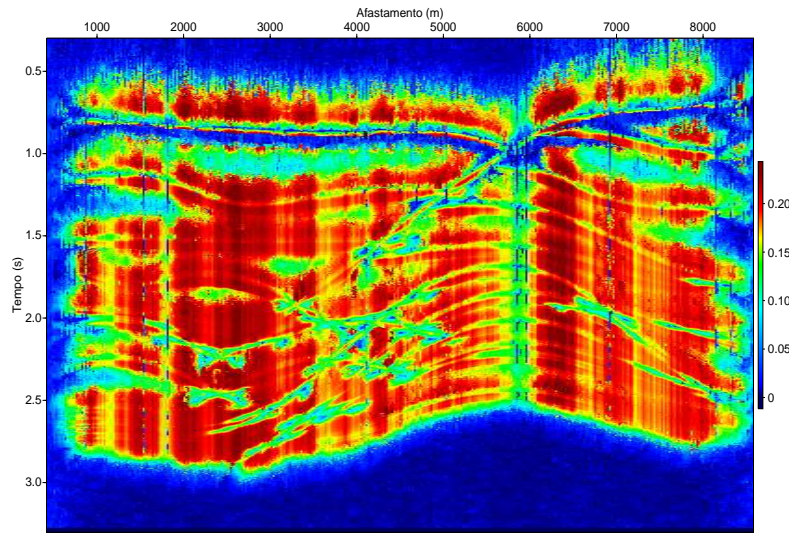

Figura 9: Seção coerência semblance resultante do empilhamento CRS-convencional.

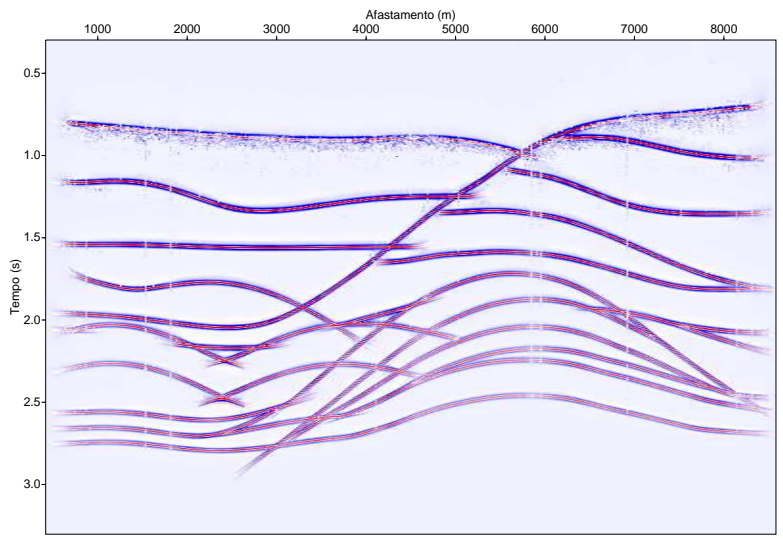

Figura 10: Seção empilhada CRS-convencional 
A figura 11 mostra o modelo de velocidade obtido após 30 iterações utilizando um modelo inicial com velocidade constante, $v_{0}=2000 \mathrm{~m} / \mathrm{s}$. Em decorrência da baixa quantidade de pontos marcados, devido à baixa qualidade da seção coerência semblance (figura 9), a inversão NIPtomográfica obteve um modelo de velocidade inferior ao da figura 8.

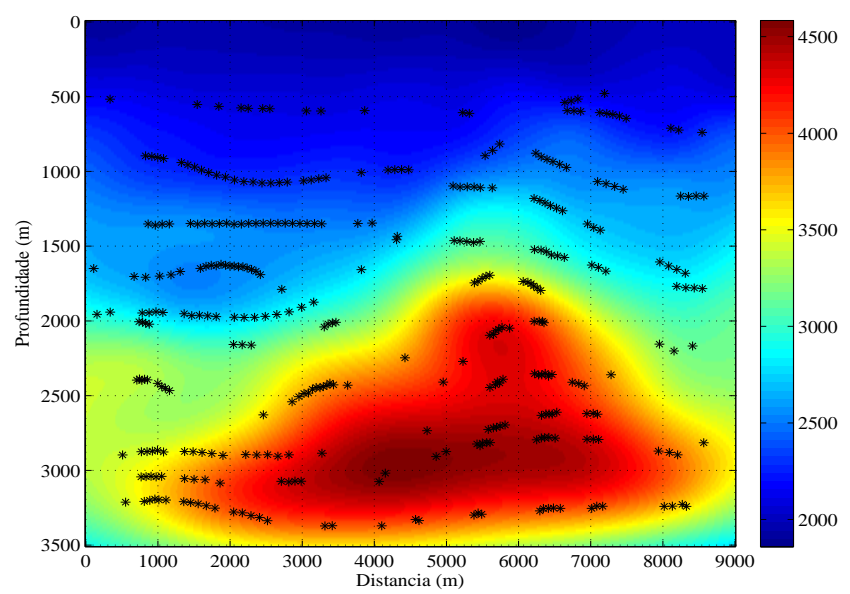

Figura 11: Modelo de velocidade resultante após 30 interações com as marcações na seção empilhada CRSoptimizada sobrepostas.

\section{Inversão: CRS-superfamília}

A terceira parte foi referente ao empilhamento CRSconvencional superfamília, e usado como entrada na inversão NIP-tomográfica para também se obter o modelo de velocidade em profundidade. Os dados de entrada ao programa de marcação automática foram as seguintes seções: a empilhada CRS-superfamília; a seção coerência semblance optimizada; a $R_{N I P}$ otimizada; a ângulo de emergência $\alpha$. O valor mínimo da coerência válida para marcação foi 0,70 , o que corresponde a mais do que o triplo nos testes anteriores. O total de pontos marcados foi de 846 , e reduzido a 830 após a edição.

A figura 12 mostra a seção coerência, onde o valor máximo está notadamente ao redor de $1,00 \mathrm{e}$, portanto, o mais alto, e servindo de medida para a alta qualidade da medida da relação $S / R$.

A figura 13 mostra a seção empilhada CRS-superfamília, que resulta numa seção com uma melhor relação $S / R$ e continuidade dos eventos em comparação às figuras $7 \mathrm{e}$ 10.

A figura 14 mostra o modelo de velocidade obtido após 30 iterações utilizando um modelo inicial com velocidade constante, $v_{0}=2000 \mathrm{~m} / \mathrm{s}$. Nota-se como a seção com uma alta coerência semblance (ver figura 12), cujo o valor máximo está ao redor de 0,9 , gerada pelo método de empilhamento CRS-parcial, aumenta a confiabilidade dos atributos CRS, e são determinantes para se obter um modelo de velocidade próximo ao modelo original.

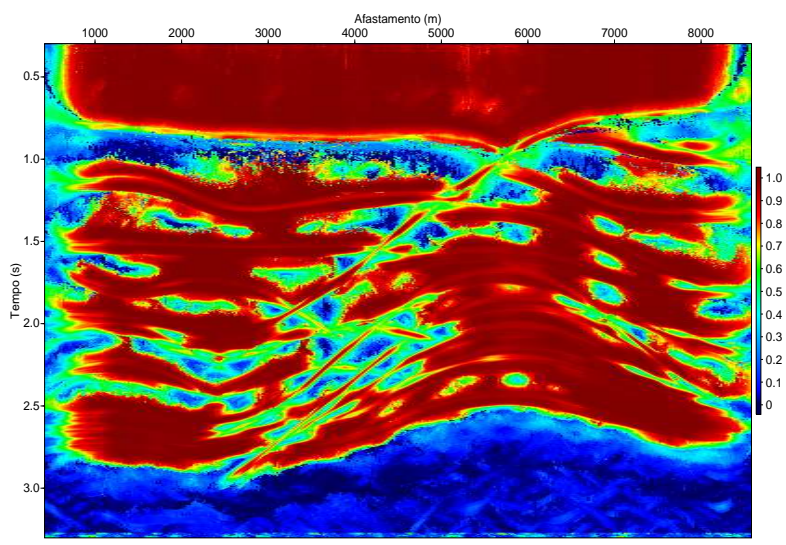

Figura 12: Seção coerência semblance resultante do empilhamento CRS-superfamília.

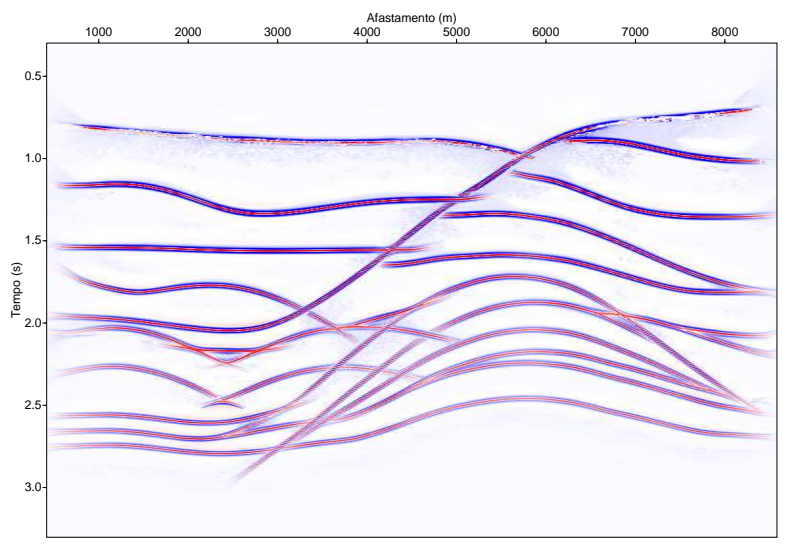

Figura 13: Seção empilhada CRS-superfamília.

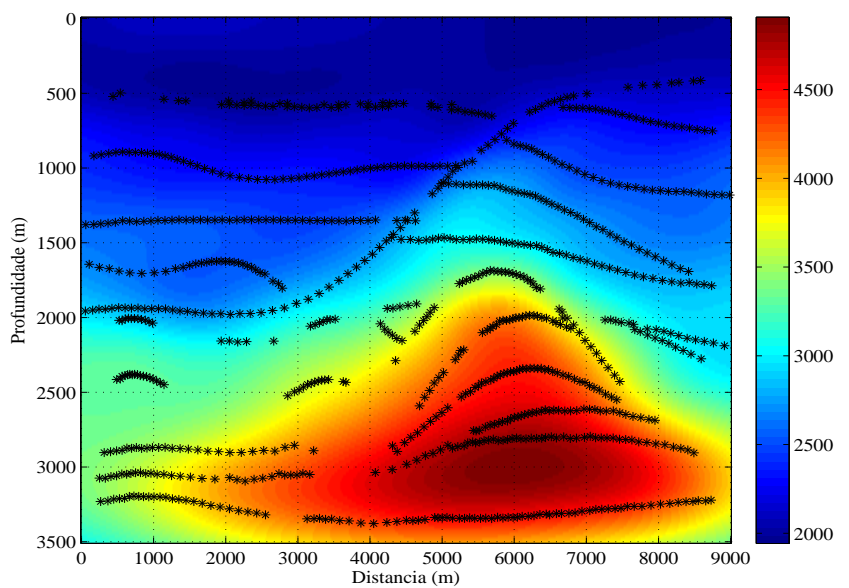

Figura 14: Modelo de velocidade resultante após 30 interações com as marcações da seção empilhada CRSsuperfamília sobrepostas.

A figura 15 mostra a maior quantidade de pontos marcados nas seções CRS-superfamília, e também a 
maior concentração de pontos em um intervalo menor de erro residual. Observa-se que se obteve um melhor ajuste dos parâmetros analisados quando utiliza-se as seções CRS-superfamílias, principalmente em relação ao parâmetro $p$.

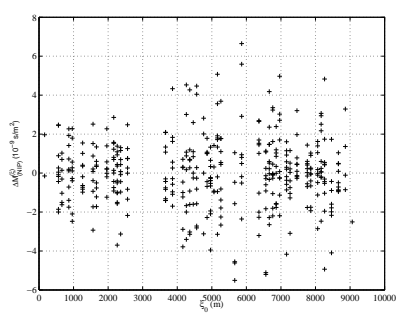

(a)

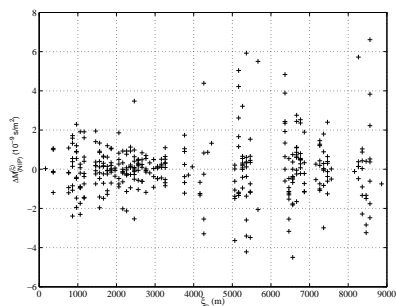

(c)

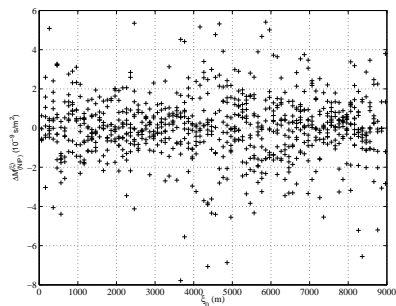

(e)

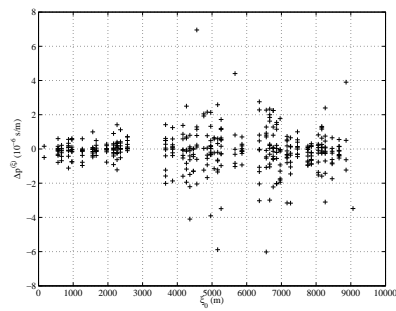

(b)

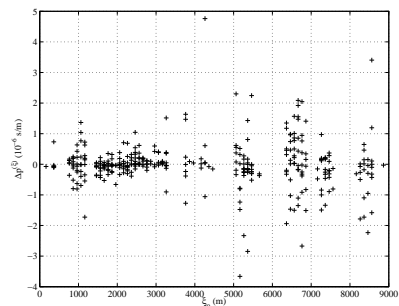

(d)

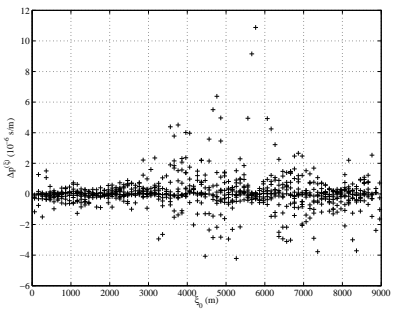

(f)
Figura 15: Resultados após 30 iterações da inversão NIPtomográfica. Erro residual da inversão para os parâmetros $M_{\text {NIP }}$ e $p$. (a) e (b) utilizando a seção CMP-automática; (c) e (d) CRS-convencional; e (e) e (f) para CRS-superfamília.

Com base nos testes realizados, concluímos que o empilhamento CRS-parcial melhora a qualidade dos dados com efeito positivo para a obtenção do modelo de velocidade com a inversão NIP-tomográfica. Neste estudo, salientamos o emprego do CRS-parcial especialmente para dados esparsos, como importante para o empilhamento e a consequente inversão NIPtomográfica.

\section{Referências}

Baykulov, M., and Gajewski, D., 2007, Prestack seismic data enhancement with crs parameters: WIT, , no. 11, 50-61.

Duveneck, E., 2004, Tomographic determination of seismic velocity models with kinematic wavefield attributes: Ph.D. thesis, Karlsruhe University, Karlsruhe.

Müller, T., 1999, The common reflection surface stack method-seismic imaging without explicit knowledge of the velocity model: Ph.D. thesis, Karlsruhe University, Karlsruhe.

Paige, C. C., and Saunders, M. A., 1982, Algorithm 583 - Isqr: Sparse linear equations and least squares problems: ACM Trans. Math. Softw., 8, no. 2, 195-209.

Pena, F., 2014, Processamento e imageamento sísmico usando o crs: Master's thesis, Universidade Federal do Pará, Belém, Pará.

\section{Agradecimentos}

Os autores agradecem às instituições brasileiras UFPA, o CNPq, e em especial ao Instituto Nacional de Ciência e Tecnologia, INCT-GP, pelo apoio ao desenvolvimento deste trabalho. Os agradecimentos são também estendidos aos parceiros do Consórcio WIT-Karlsruhe-Hamburgo, Alemanha, pela cooperação acadêmica. 